\title{
Human adipose tissue-derived mesenchymal stromal cells promote B-cell motility and chemoattraction
}

\author{
LAURA BARRIO ${ }^{1}$, VICTOR DELGADO CUEVAS ${ }^{1}$, RAMÓN MENTA ${ }^{2}$, \\ PABLO MANCHEÑO-CORVO ${ }^{2}$, OLGA DELAROSA ${ }^{2}$, WILFRIED DALEMANS ${ }^{3}$, \\ ELEUTERIO LOMBARDO ${ }^{2, *} \&$ YOLANDA R. CARRASCO ${ }^{1, *}$ \\ ${ }^{1}$ B Cell Dynamics Laboratory, Department of Immunology and Oncology, Centro Nacional de Biotecnología (CNB)- \\ CSIC, UAM-Campus Cantoblanco, Madrid, Spain, and ${ }^{2}$ TiGenix SAU, Parque Tecnológico de Madrid, Madrid, \\ Spain, ${ }^{3}$ TiGenix NV, Leuven, Belgium
}

\begin{abstract}
Background aims. Mesenchymal stromal cells hold special interest for cell-based therapy because of their tissue-regenerative and immunosuppressive abilities. B-cell involvement in chronic inflammatory and autoimmune pathologies makes them a desirable target for cell-based therapy. Mesenchymal stromal cells are able to regulate B-cell function; although the mechanisms are little known, they imply cell-to-cell contact. Methods. We studied the ability of human adipose tissue-derived mesenchymal stromal cells (ASCs) to attract B cells. Results. We show that ASCs promote B-cell migration through the secretion of chemotactic factors. Inflammatory/innate signals do not modify ASC capacity to mediate B-cell motility and chemotaxis. Analysis of a panel of B cell-related chemokines showed that none of them appeared to be responsible for B-cell motility. Other ASC-secreted factors able to promote cell motility and chemotaxis, such as the cytokine interleukin-8 and prostaglandin $\mathrm{E}_{2}$, did not appear to be implicated. Conclusions. We propose that ASC promotion of B-cell migration by undefined secreted factors is crucial for ASC regulation of B-cell responses.
\end{abstract}

Key Words: $B$ cells, chemotaxis, mesenchymal stromal cells, migration

\section{Introduction}

Mesenchymal stromal cells (MSCs) are multipotent adult stem cells able to differentiate into distinct cell types. They are present in practically all tissues and are a key element in tissue repair and regeneration (1). In addition to their differentiation potential, MSCs can modulate the function of several immune cell types through secretion of soluble factors and/or cell contact-dependent mechanisms $(1,2)$. This feature makes them an attractive potential tool for cell-based therapy for chronic inflammatory and autoimmune pathology. The MSC-mediated immunosuppressive effects on $\mathrm{T}$-cell responses are well established $(3,4)$; there is also evidence that they inhibit natural killer cell function (5), regulate dendritic cell maturation and trafficking $(6,7)$ and modulate macrophage differentiation (8). In contrast, little is known of the MSC effect on B-cell function.
B cells are central to the adaptive immune response; they specialize in antigen recognition and the production of highly specific antibodies. They also have an important role as antigen-presenting cells for $\mathrm{T}$ cells and regulate immune responses through interleukin (IL)-10 secretion. Nevertheless, B cells participate in the development of numerous autoimmune disorders (9-11) and are crucial mediators in allograft rejection (12). The beneficial effects of B-cell-depleting therapies on chronic inflammatory (Crohn's disease) and autoimmune pathologies (rheumatoid arthritis) stress the relevance of targeting B cells for immunosuppression in such diseases. Some reports point to a suppressive effect of MSCs on antigen-triggered $\mathrm{B}$-cell activation and differentiation (13-18). The extent of B-cell suppression appears to depend on the MSC source (15) and the need for B cell-MSC contact $(14,18)$. We studied the ability of human MSCs derived from adipose tissue (ASCs) to

*These authors contributed equally to this work.

Correspondence: Yolanda R. Carrasco, PhD, B Cell Dynamics Laboratory, Department of Immunology and Oncology, Centro Nacional de Biotecnología (CNB)-CSIC, Darwin 3, UAM-Campus Cantoblanco, Madrid E-28049, Spain. E-mail: ycarrasco@cnb.csic.es 
promote $\mathrm{B}$-cell migration and chemoattraction to facilitate ASC-B-cell contact.

\section{Methods \\ Cell isolation and culture}

Human adult MSCs were obtained from adipose tissue of 3 donors (donors 10, 14 and 16), as described (19). Briefly, lipoaspirates of human adipose tissue from healthy donors were washed with phosphate-buffered saline (PBS), digested with $0.075 \%$ collagenase type-I (Invitrogen, Camarillo, $\mathrm{CA}$, USA; $37^{\circ} \mathrm{C}, 30 \mathrm{~min}$ ), washed with $\mathrm{PBS} / 10 \%$ fetal bovine serum (FBS) and treated with ammonium chloride to eliminate residual erythrocytes. Cells were cultured in Dulbecco's modified Eagle's medium $/ 10 \%$ FBS and passaged when they reached $90 \%$ confluence. Cells were characterized phenotypically, as described (19). ASCs from donors 10, 14 and 16 were used at population doubling (pd) 14, pd 12 and pd 14, respectively. Primary human foreskin fibroblasts were obtained from a healthy donor and expanded in vitro. The human endothelial cell line Eahy derived from human umbilical vein endothelial cells was kindly provided by Dr J. Millán (Centro de Biología Molecular Severo Ochoa, Madrid). The human fibrosarcoma cell line HT1080 from the American Type Culture Collection was kindly provided by Dr J.M. Rodríguez-Frade (CNBCSIC, Madrid).

Human B cells were obtained from buffy coats of healthy donors, kindly provided by the National Transfusion Centre of the Comunidad Autonoma de Madrid. Briefly, each blood sample was diluted 1:1 in PBS and centrifuged on a Ficoll density gradient (GE Healthcare Madrid, Spain; $1800 \mathrm{rpm}, 30 \mathrm{~min}$, $25^{\circ} \mathrm{C}$ ). The peripheral blood mononuclear cell interface was recovered, washed in Roswell Park Memorial Institute medium (RPMI) $/ 10 \%$ FBS, and the B-cell population was purified with the use of the Dynabeads Untouched Human B cells isolation kit (Invitrogen); purity was confirmed by CD19 staining and flow cytometry ( $\geq 98 \%)$.

Experimentation with human tissue samples was approved by the CNB-CSIC Bioethics Committee and conforms to institutional, national and European Union regulations.

\section{Flow cytometry analysis}

Human B cells $\left(2 \times 10^{5}\right)$ were stained with the use of fluorescently labeled antibodies to CD19, CD27, immunoglobulin (Ig)M, IgD, C-X-C-motif chemokine receptor 4 (CXCR4) and C-C-motif chemokine receptor 2 (CCR2) (BioLegend, San Diego, CA,
USA) or with antibodies to CXCR1, CXCR2 (Biosource, Invitrogen) and CXCR3 (R\&D Systems, Minneapolis, MN, USA), followed by fluorescently labeled $\mathrm{Fab}$ anti-mouse IgG (Jackson Immunoresearch Labs, West Grove, PA, USA) in PBS $/ 1 \%$ bovine serum albumin $/ 1 \%$ FBS $\left(20 \mathrm{~min}, 4^{\circ} \mathrm{C}\right)$; B cells were washed and analyzed in a FACScalibur flow cytometer (BD Biosciences, San Jose, CA, USA).

\section{$B$-cell migration assays}

ASCs $\left(3 \times 10^{4}\right)$ were seeded in p24 multiwell plates $(24 \mathrm{~h})$, and stimuli were added (interferon [IFN]- $\gamma$, $30 \mathrm{ng} / \mathrm{mL}$, Peprotech (London, UK); the Toll-like receptor [TLR]4 ligand lipopolysacharide [LPS], from Salmonella typhymurium, $0.1 \mu \mathrm{g} / \mathrm{mL}$, List Biological Laboratories (Campbell, CA, USA); the TLR3 ligand poly I:C, $0.1 \mu \mathrm{g} / \mathrm{mL}$, Invivogen, San Diego, CA, USA) in a final volume of $500 \mu \mathrm{L} \mathrm{RPMI} /$ $10 \%$ FBS per well. Supernatants were collected $72 \mathrm{~h}$ after stimulation and used immediately in the B-cell migration assay. Eahy cells, primary fibroblasts and HT1080 cells $\left(3 \times 10^{4}\right)$, were seeded in p24 multiwell plates in a final volume of $500 \mu \mathrm{L} \mathrm{RPMI} / 10 \%$ FBS per well; supernatants were collected at $72 \mathrm{~h}$ and used immediately in the B-cell migration assay.

Freshly purified B cells $\left(3 \times 10^{5}\right)$ were loaded into the top insert of Boyden chambers $(5-\mu \mathrm{m}$ pore size, Corning, Tewksbury, MA, USA) in a final volume of $100 \mu \mathrm{L} \mathrm{RPMI} / 10 \% \mathrm{FBS}$; the bottom well was filled with $600 \mu \mathrm{L}$ supernatant (obtained from ASCs, Eahy, fibroblast or HT1080 cell culture), $\mathrm{RPMI} / 10 \%$ FBS alone or with recombinant human C-X-C-motif ligand 12 (CXCL12), C-C-motif ligand 2 (CCL2), CXCL8, CXCL10 (Peprotech) or prostaglandin $\mathrm{E}_{2}\left(\mathrm{PGE}_{2}\right.$; Sigma-Aldrich, St Louis, MO, USA) at specified concentrations, in duplicate or triplicate. In assays with CCL2, the top insert was precoated with fibronectin $\left(20 \mu \mathrm{g} / \mathrm{mL}, 1 \mathrm{~h}, 37^{\circ} \mathrm{C}\right.$; Sigma) to facilitate B-cell migration. Plates were incubated for $3 \mathrm{~h}\left(37^{\circ} \mathrm{C}, 5 \% \mathrm{CO}_{2}\right)$. Where indicated, $\mathrm{B}$ cells were pre-incubated with anti-CXCR4 blocking antibody (clone 12G5, BioLegend; $10 \mu \mathrm{g} / \mathrm{mL}, 20$ min, $37^{\circ} \mathrm{C}$, with shaking) or with the isotype control (mouse IgG2a, BioLegend) and loaded into the Boyden chamber; the antibodies remained present throughout the assay. B cells that migrated to the bottom well were counted for $1 \mathrm{~min}$ at high flow rate in the flow cytometer; B-cell input was estimated similarly, with $3 \times 10^{5} \mathrm{~B}$ cells added to $600 \mu \mathrm{L}$ RPMI $/ 10 \%$ FBS. Migration frequency in each condition was calculated as the ratio between the number of B cells in the bottom well and total B-cell input and multiplied by 100 . 


\section{Reverse transcriptase-polymerase chain reaction}

ASCs $\left(1.5 \times 10^{5}\right)$ were seeded in p6 multiwell plates $(24 \mathrm{~h})$ and were then stimulated (IFN- $\gamma$, LPS, poly I:C). Cells were recovered $24 \mathrm{~h}$ after stimulation and lysed for RNA extraction with the use of Trizol reagent (Invitrogen). Messenger RNA (mRNA) levels of the indicated chemokines were detected by means of reverse transcriptase-polymerase chain reaction (RT-PCR), with the use of primers specific for each chemokine; mRNA amplification of the housekeeping gene $\beta$-actin was used as control.

\section{Measurement of secreted chemokine levels and other factors}

ASCs were seeded in p6 multiwell plates; after $24 \mathrm{~h}$, stimuli were added (IFN- $\gamma$, LPS, poly I:C) in $2 \mathrm{~mL}$ final volume. Supernatants were collected 24, 48 and $72 \mathrm{~h}$ after stimulation and stored at $-80^{\circ} \mathrm{C}$. Levels of secreted CXCL12 and CCL2 were measured with the use of the Luminex-based MilliPlex Human cytokine/chemokine immunoassay (Millipore, Temecula, CA, USA). An enzyme-linked immunoassay (ELISA) was used to measure CXCL8 (eBioscience, San Diego, CA, USA) and $\mathrm{PGE}_{2}$ (Thermo Scientific, Waltham, MA, USA) secretion in ASC supernatants collected at $48 \mathrm{~h}$ of culture.

\section{Statistical analysis}

Statistical data analysis to compare groups of 2 conditions was performed by applying the 2-tailed Student's $t$-test with the use of GraphPad Prism v. 4.0b. A value of $P \leq 0.05$ was considered statistically significant.

\section{Results}

ASCs promote B-cell migration and chemoattraction

To evaluate the ability of ASCs to promote B-cell migration, we cultured ASCs from 3 donors in complete medium for $72 \mathrm{~h}$; we then collected the supernatants and assessed their B-cell chemotactic activity in Boyden chambers. We obtained B cells from peripheral blood samples of healthy donors. The freshly isolated B-cell population $\left(\mathrm{CD} 19^{+}\right.$cells, $>98 \%$ purity) usually contained $\sim 70 \%$ mature naive $\mathrm{B}$ cells $\left(\mathrm{CD} 19^{+} \mathrm{CD}_{27}^{-} \mathrm{IgM}^{+} \mathrm{IgD}^{+}\right)$and $\sim 30 \%$ memory B cells $\left(\mathrm{CD} 19^{+} \mathrm{CD} 27^{+}\right.$) (Figure $\left.1 \mathrm{~A}\right)$. Purified $\mathrm{B}$ cells were allowed to migrate in complete medium alone or in ASC supernatants $\left(3 \mathrm{~h}, 37^{\circ} \mathrm{C}\right)$. With the use of flow cytometry, we estimated the fraction of $\mathrm{B}$ cells that migrated in each condition. We found that ASC supernatants promoted B-cell
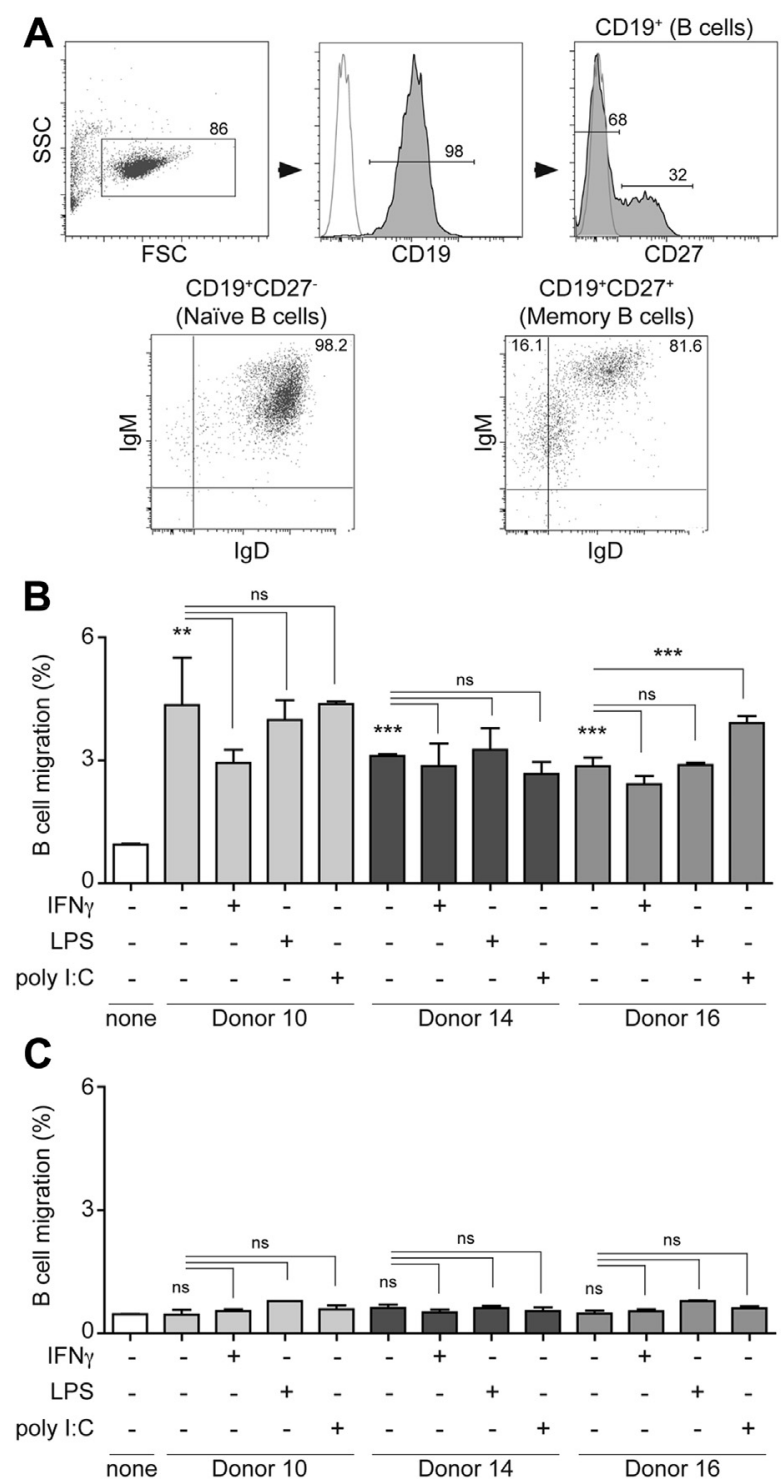

Figure 1. ASCs secrete factors that promote B-cell migration. (A) Representative profiles of side scatter (SSC), forward scatter (FSC), CD19 and CD27 expression in the B-cell population isolated from human peripheral blood of healthy donors. Bottom: Dot plots of IgM and IgD expression for the naive B-cell (gated as $\mathrm{CD} 19^{+} \mathrm{CD} 27^{-}$) and memory B-cell fractions (gated as $\mathrm{CD} 19^{+} \mathrm{CD} 27^{+}$). Percentages of each gated population are indicated; gray line in CD19 and CD27 profiles, isotype control. (B) Migration frequency of B cells in medium alone or in supernatants from ASCs, unstimulated or stimulated with IFN $-\gamma$, LPS or poly I:C (72 h); ASCs isolated from 3 donors (donors 10, 14 and 16) were used. (C) As in (B), with the use of ASC-derived supernatants diluted $1: 10$ in $\mathrm{RPMI} / 10 \%$ FBS. Data in (B, C) show mean $\pm \mathrm{SD}$ of 1 representative experiment $(n=4)$. Statistical significance is indicated for each case in $(\mathrm{B}, \mathrm{C}) .{ }^{* *} P<0.001 ;{ }^{* * *} P<$ 0.0001 ; ns, not significant.

chemotaxis, with a 3- to 5-fold increase compared with basal B-cell migration frequencies (medium alone) (Figure 1B). B-cell chemotactic activity was similar for the distinct ASC donor supernatants (Figure 1B) and was lost when supernatants were diluted 1:10 (Figure 1C). 
To determine whether inflammatory and/or innate stimulation of ASCs regulates their ability to promote B-cell migration, we cultured ASCs with various stimuli $(72 \mathrm{~h})$ and collected the supernatants. We used the cytokine IFN- $\gamma$ and the TLR ligands LPS and poly I:C as inflammatory/innate signals. Migration assays were performed as above, with the use of the ASC-stimulated supernatants. We found no significant modification in ASC-mediated B-cell chemotaxis caused by stimulation compared with unstimulated ASCs (Figure 1B). The supernatants lost the ability to promote $\mathrm{B}$-cell migration when diluted 10-fold (Figure 1C).

We compared ASC capacity to attract B cells with that of other cell types of mesoderm or mesenchymal origin. With the use of supernatants of endothelial cells (Eahy; mesodermal cell type), fibroblasts and the fibrosarcoma cell line HT1080 (both mesenchymal cell types) after 72-h culture in complete medium, we assessed their B-cell chemotactic activity in Boyden chambers, with ASC supernatant from 1 donor as positive control. These other cell types promoted B-cell migration by secreting factors to the medium, with a 2- to 2.5-fold increase compared with basal B-cell migration frequencies (Supplementary Figure 1). Frequency values were nonetheless lower for mesenchymal cell-derived medium than for those obtained with ASC supernatant (Supplementary Figure 1).

The data thus showed that ASCs can promote $\mathrm{B}$-cell migration and attract $\mathrm{B}$ cells; this effect is mediated by a factor(s) secreted by ASCs to the medium. Stimulation of ASCs with classic inflammatory/innate signals does not modify this ability. ASC capacity to attract B cells is greater than that of other mesenchymal cell types.

\section{ASCs produce distinct chemokines}

To identify the ASC-secreted factor(s) responsible for B-cell migration, we analyzed the mRNA expression of a panel of chemokines, including the homeostatic chemokines CXCL12, CXCL13, CCL19 and CCL21, all involved in B-cell homing and location in secondary lymphoid organs $(20,21)$. We also evaluated mRNA expression of several inflammatory chemokines (CCL2, CCL5, CCL20) implicated in lymphocyte trafficking and migration in inflammatory conditions $(21-23)$. ASCs from the 3 donors were cultured alone or with stimulation (IFN- $\gamma$, LPS, poly I:C) for $24 \mathrm{~h}$, processed, and chemokine expression analyzed by means of RTPCR. All donors in all conditions expressed CXCL12, but none of the other homeostatic chemokines studied (Figure 2A). For the inflammatory chemokines, CCL2 data were similar to CXCL12;
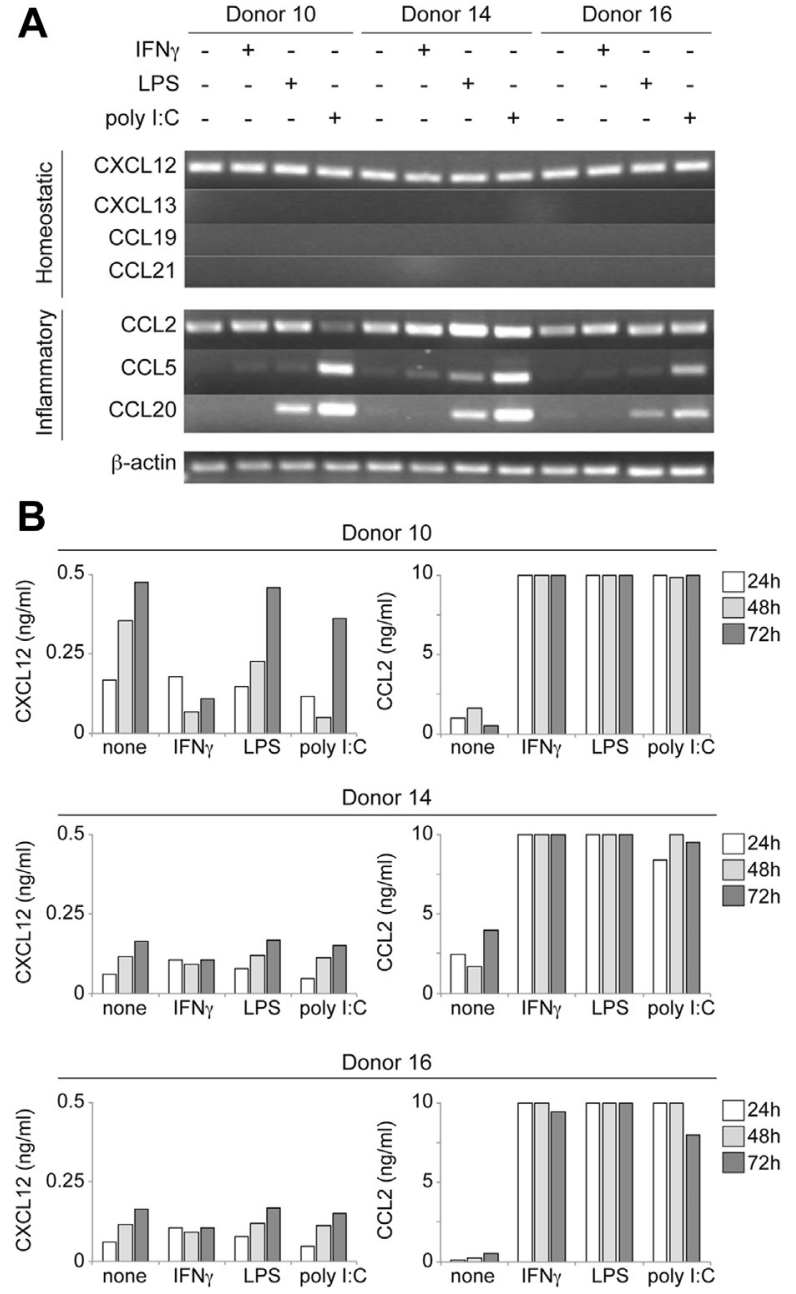

Figure 2. ASCs express and secrete distinct chemokines. (A) RT-PCR analysis of mRNA expression of the homeostatic chemokines CXCL12, CXCL13, CCL19 and CCL21 and the inflammatory chemokines CCL2, CCL5 and CCL20, in ASCs cultured alone or with indicated stimuli $(24 \mathrm{~h})$. ASCs from 3 donors (donors 10, 14 and 16) were studied; $\beta$-actin mRNA amplification was used as control of expression. (B) Luminex assay data show secreted CXCL12 (left) and CCL2 levels (right) in supernatants from all 3 ASC donors, cultured alone or with indicated stimuli for the times specified. Data in (A, B) show 1 representative experiment $(n=3)$.

CCL5 and CCL20 were barely detectable in unstimulated ASCs, and their levels increased after inflammatory/innate ASC stimulation (Figure 2A). Results were comparable among the 3 donors.

Because the B-cell chemotactic abilities of ASCs were similar, independent of the presence of inflammatory/innate stimuli (Figure 1B), CXCL12 and/or CCL2 were candidates as mediators of B-cell migration (Figure 2A). We used Luminex-based immunoassays to measure secreted CXCL12 and CCL2 levels in ASC supernatants from the 3 donors, cultured alone or with stimuli and collected at several time points (24, 48 and $72 \mathrm{~h}$ ). All 3 donors showed low levels of CXCL12 secretion and accumulation 
with time $(\sim 0.2-0.5 \mathrm{ng} / \mathrm{mL}$; equivalent to $\sim 0.05$ $\mathrm{nmol} / \mathrm{L}$ ) (Figure 2B), with no changes caused by inflammatory stimuli. Secreted CCL2 levels were $\sim 1 \mathrm{ng} / \mathrm{mL}$ (equivalent to $\sim 0.1 \mathrm{nmol} / \mathrm{L}$ ) in unstimulated ASCs, which increased at least 10-fold in stimulated ASCs $(\geq 10 \mathrm{ng} / \mathrm{mL}$; equivalent to $\sim 1$ $\mathrm{nmol} / \mathrm{L}$ ) (Figure 2B).

The data thus indicated that ASCs from distinct donors expressed and secreted detectable levels of CXCL12 and CCL2. Inflammatory signals increased CCL2, CCL5 and CCL20 expression in ASC.

\section{CXCL12 and CCL2 do not mediate ASC chemoattraction of $B$ cells}

We studied the migratory response of peripheral blood B cells to CXCL12 and CCL2. With the use of flow cytometry, we analyzed the expression of their respective receptors, CXCR4 and CCR2, at the B-cell surface. Both CXCR4 and CCR2 displayed a homogeneous expression pattern in the peripheral blood B-cell population (Figure 3A,B). We evaluated the migratory response of $\mathrm{B}$ cells to distinct gradients of recombinant CXCL12 or CCL2 in Boyden chambers. Freshly isolated peripheral blood B cells migrated in response to CXCL12 gradients, with a maximum at $10 \mathrm{nmol} / \mathrm{L}$ chemokine (Figure $3 \mathrm{~A}$ ). $\mathrm{B}$ cells did not migrate in response to CCL2 in any condition, even when fibronectin was added to facilitate cell motility (Figure 3B).

The results suggested that CCL2 was not responsible for ASC promotion of B-cell motility. We interfered with CXCR4 function to evaluate the role of CXCL12. When B cells were pre-incubated with a CXCR4-blocking antibody, which was maintained in the Boyden chamber throughout the assay, CXCL12mediated B-cell migration decreased by half (Figure 3C). In migration assays with the use of supernatants from unstimulated and stimulated ASC cultures of 2 donors $(72 \mathrm{~h}$ ), alone or with the CXCR4blocking antibody, there was no decrease in the B-cell chemotactic response when we interfered with CXCR4 function (Figure 3D,E). The data thus indicated that ASC-derived CXCL12 is not the secreted factor responsible for ASC-mediated B-cell chemotaxis; other ASC-expressed factor(s) might be involved.

\section{ASCs produce other factors implicated in cell motility and chemotaxis}

We studied the role of other ASC-produced factors that might be involved in promoting B-cell motility and attraction. ASCs secrete B-cell activating factor
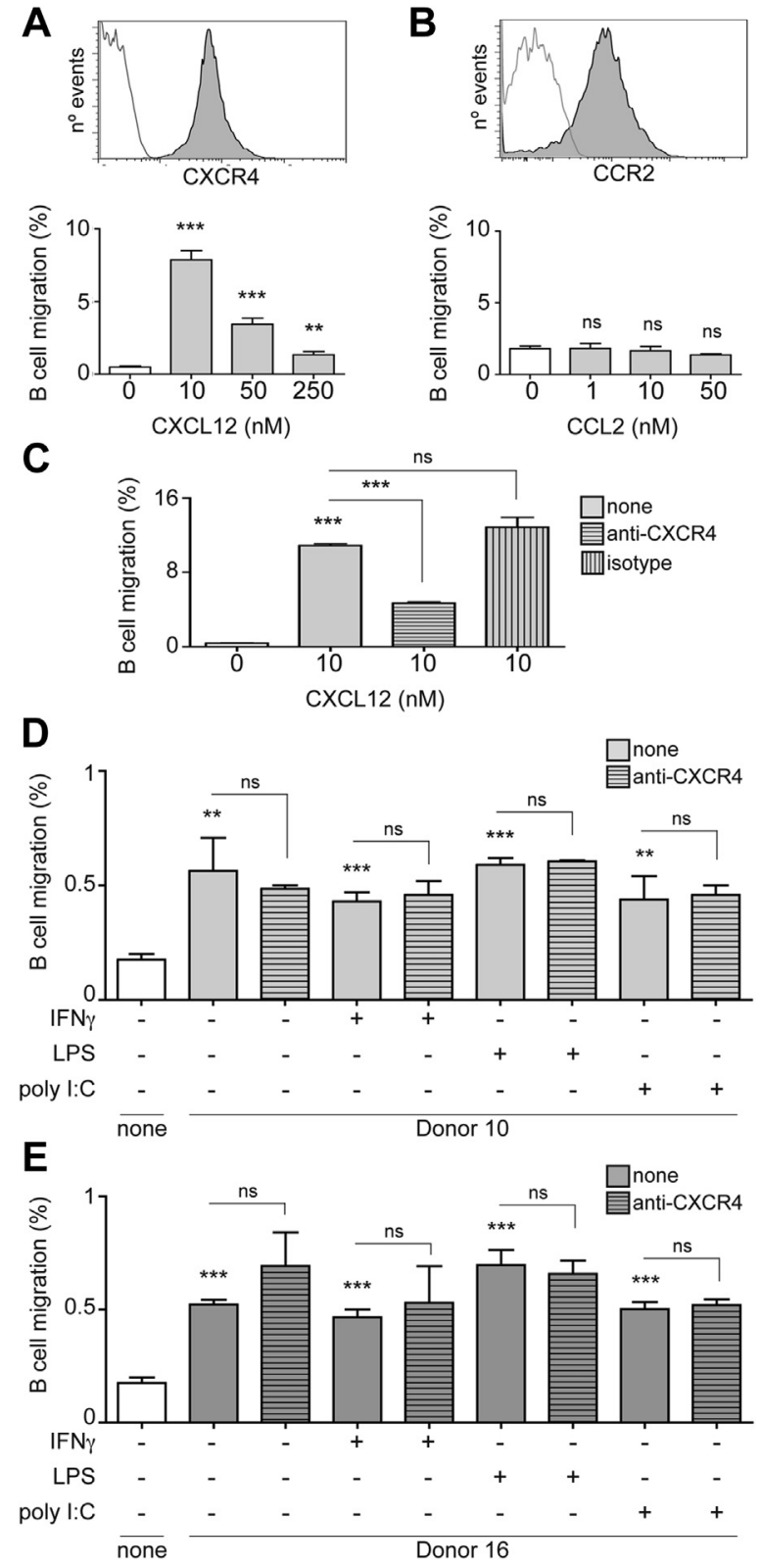

Figure 3. CXCL12 and CCL2 are not involved in ASC promotion of B-cell migration. (A) Top: Representative profile of CXCR4 expression in peripheral blood B cells; gray line, isotype control. Bottom: B-cell migration frequency in response to distinct CXCL12 doses $(0,10,50$ and $250 \mathrm{nmol} / \mathrm{L})$. (B) Top: Representative profile of CCR2 expression in peripheral blood B cells; gray line, isotype control. Bottom: B-cell migration frequency in response to distinct CCL2 doses $(0,1,10$ and $50 \mathrm{nmol} / \mathrm{L})$. (C) Migration frequency of B cells in response to CXCL12 $(10 \mathrm{nmol} / \mathrm{L})$; B cells were untreated (none), treated with anti-CXCR4 blocking antibody (anti-CXCR4) or with the isotype control (isotype). (D) Migration frequency of $\mathrm{B}$ cells, untreated (none) or treated with anti-CXCR4 blocking antibody (anti-CXCR4), in response to supernatants from ASC donor 10 cultured alone or with indicated stimuli $(72 \mathrm{~h})$. (E) As in (D), supernatants derived from ASC donor 16 are used. Data show mean \pm SD of 1 representative experiment (for $\mathrm{A}, \mathrm{B}$ and $\mathrm{C}, n=3$; for $\mathrm{D}$ and $\mathrm{E}, n=2$ ). Statistical significance is indicated for each case. ${ }^{*} P<0.001$; ***P $<0.0001 ;$ ns, not significant. 
(BAFF) and a proliferation-inducing ligand, 2 cytokines of the tumor necrosis factor family that are associated with B-cell survival, proliferation and maturation (24). Whereas BAFF exposure enhances the human B-cell chemotactic response to certain chemokines, neither BAFF nor APRIL alone appears to induce B-cell chemotaxis (25). ASCs secrete the cytokine IL-8 and the lipid $\mathrm{PGE}_{2}(19,26)$. IL-8, also known as CXCL8, has strong chemotactic activity in neutrophils and $\mathrm{T}$ cells and signals through its receptors CXCR1 and CXCR2 (27). PGE 2 belongs to a group of bioactive lipid mediators that regulate several biological processes (28); prostaglandins can also regulate cell migration and act as chemotactic agents (29-31).

In the ELISA, we detected the cytokine IL-8 (0.2 $\mathrm{ng} / \mathrm{mL} ; \sim 0.05 \mathrm{nmol} / \mathrm{L})$ and the lipid $\mathrm{PGE}_{2}(0.2 \mathrm{ng} /$ $\mathrm{mL} ; \sim 1 \mathrm{nmol} / \mathrm{L}$ ) in ASC supernatants (Figure $4 \mathrm{~A}$ ). We evaluated the ability of CXCL8 and $\mathrm{PGE}_{2}$ to promote $\mathrm{B}$-cell migration. Although human $\mathrm{B}$ cells express the $4 \mathrm{PGE}_{2}$ receptors (EP1-EP4) (28), we detected no chemotactic response to distinct $\mathrm{PGE}_{2}$ gradients (Figure 4B). We evaluated surface expression of CXCR1 and CXCR2 in human B cells isolated from peripheral blood; these cells expressed low CXCR1 levels, and CXCR2 was undetectable (Figure 4C). In Boyden chambers, we observed a moderate B-cell response only in the presence of 100 $\mathrm{nmol} / \mathrm{L}$ CXCL8.

Mouse bone marrow-derived MSCs promote T-cell migration and attraction by producing CXCL9 and CXCL10 and signaling through the CXCR3 receptor expressed at the T-cell surface (32). Human peripheral blood B cells expressed CXCR3 (Figure 4E). We analyzed the chemotactic response of $\mathrm{B}$ cells to gradients of CXCL10, which had a higher affinity for its receptor than CXCL9. We detected a small migration increase only at the highest CXCL10 dose used (100 nmol/L; Figure 4F).

To evaluate whether an additive or synergistic effect of the factors in ASC supernatants was responsible for $\mathrm{B}$-cell migration, we exposed $\mathrm{B}$ cells to gradients of a combination of $\mathrm{PGE}_{2}(1 \mathrm{nmol} / \mathrm{L})$ and CXCL8 (0.1 nmol/L), alone or with CXCL12 $(0.1 \mathrm{nmol} /)$, at doses similar to those measured in the ASC supernatants; we included CXCL10 in 1 mixture. None of the combinations tested induced B-cell migration above basal values (Figure 4G).

ASCs produce CXCL8 and $\mathrm{PGE}_{2}$, both of which can regulate and promote cell migration. Human peripheral blood B cells did not respond to either of them in chemotaxis assays, alone, combined or in the presence of CXCL12. CXCL10 has poor chemotactic activity for human $\mathrm{B}$ cells, and its combination with CXCL8 and $\mathrm{PGE}_{2}$ did not enhance B-cell migration.
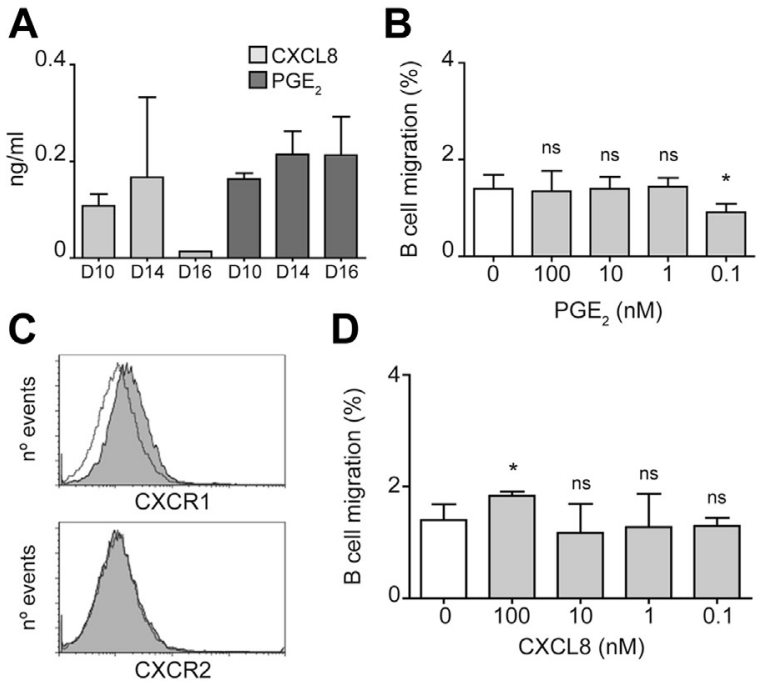

D
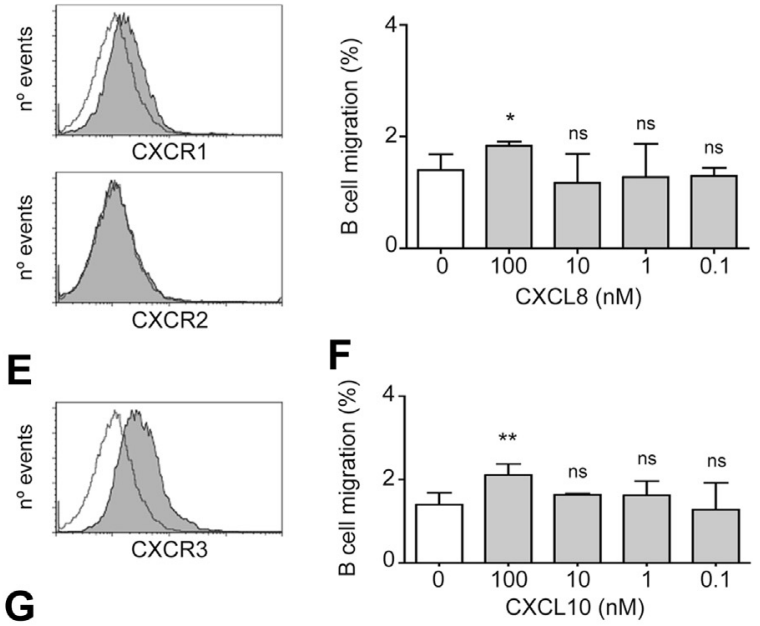

G

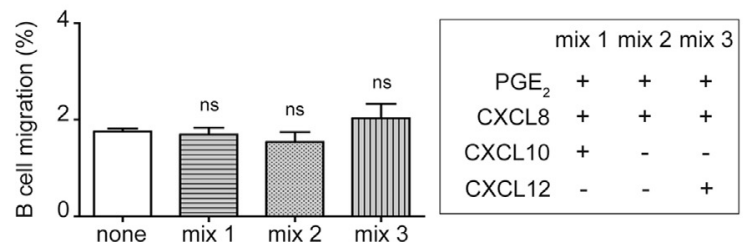

Figure 4. ASC-induced B-cell chemotaxis does not involve CXCL8, CXCL10 or $\mathrm{PGE}_{2}$. (A) CXCL8 and $\mathrm{PGE}_{2}$ levels measured by means of ELISA in supernatants of ASCs from 3 donors (donor 10, D10; donor 14, D14; donor 16, D16), collected after 48-h culture. (B) B-cell migration frequency in response to distinct $\mathrm{PGE}_{2}$ doses. (C) Representative profile of CXCR1 (top) and CXCR2 expression (bottom) in peripheral blood B cells; gray line, isotype control. (D) B-cell migration frequency in response to distinct CXCL8 doses. (E) Representative profile of CXCR3 expression in peripheral blood B cells; gray line, isotype control. (F) B-cell migration frequency in response to distinct CXCL10 doses. (G) Migration frequency of B cells exposed to medium alone (none) or to distinct mixtures of $\mathrm{PGE}_{2}(1 \mathrm{nmol} / \mathrm{L}), \mathrm{CXCL} 8$ $(0.1 \mathrm{nmol} / \mathrm{L}), \operatorname{CXCL} 10(1 \mathrm{nmol} / \mathrm{L})$ and $\operatorname{CXCL} 12(0.1 \mathrm{nmol} / \mathrm{L})$, specified in the table (right). Data in (A) show mean \pm SD of 2 independent experiments. Data in (B, D, F and G) show mean \pm $\mathrm{SD}$ of triplicates from 1 representative experiment $(\mathrm{n}=3)$. Statistical significance is indicated for each case (B, D, F and G). ${ }^{*} P<$ $0.05 ; * P<0.001 ;$ ns, not significant.

\section{Discussion}

MSCs modulate immune system function. They suppress $\mathrm{T}$-cell responses through the local secretion of factors such as nitric oxide in mice, or indoleamine 2,3-dioxygenase and $\mathrm{PGE}_{2}$ in humans $(2,3)$. These MSC-derived factors act in concert with MSC-produced chemokines, which facilitate T-cell migration and MSC:T-cell contact (32). The 
ability of MSCs to promote T-cell motility and chemoattraction is thus critical for their T-cell immunosuppressive properties. It is less well known that MSC also inhibit antigen-triggered B-cell proliferation and plasma cell differentiation $(13,16-18,33)$. The mechanisms are little studied but appear to require B-cell proximity to MSCs. Our study shows the ability of human ASCs to promote B-cell motility and chemotaxis by the secretion of factors, possibly chemokines. We confirmed these results with the use of ASCs from 3 different donors. We analyzed ASC expression of various homeostatic and inflammatory chemokines; none appeared to be responsible for the B-cell chemotactic response. ASCs produce CXCL8 and the lipid PGE $_{2}(19,26)$, both able to regulate and promote cell motility (27,29-31); their B-cell chemotactic activity, alone or combined, was undetectable at the doses secreted by ASCs. We propose that ASC ability to attract B cells is crucial for modulation of B-cell responses, although further studies are needed to identify the factor(s) implicated in this attraction.

We found poor CXCL8 and CXCL10 chemotactic activity in human peripheral blood B cells, which coincided with previous findings $(34,35)$. CXCL8 production by ASCs nonetheless implied the ability to attract other immune cells with robust chemotactic responses to this chemokine and possibly to modulate their function. Neutrophils respond strongly to low CXCL8 doses and have critical functions in inflammation and innate immune responses (27); study of the ASC-neutrophil axis might be revealing, relative to ASC use for cell-based therapy. $\mathrm{PGE}_{2}$ production by ASCs was not involved in B-cell attraction. Prostanoids are part of a complex regulatory network that regulates immune functions and the surrounding microenvironment (28). In B cells, $\mathrm{PGE}_{2}$ shifts the pleiotropic actions of IL-21, promoting IL21-mediated B-cell death (36). $\mathrm{PGE}_{2}$ produced by MSCs mediates immunosuppression of $\mathrm{T}$ cells and natural killer cells (2), which suggests that it is a good candidate for B-cell function modulation by ASCs.

It has been proposed that MSCs are not intrinsically immunosuppressive but need local stimulation by inflammatory mediators at the site of tissue damage $(2,3)$. Inflammatory signals induce MSC production of $\mathrm{T}$-cell-attracting chemokines and increase expression of adhesion molecules at the MSC surface to facilitate T-cell adhesion (32). Our results show that in vitro stimulation of human ASCs with inflammatory mediators such as IFN- $\gamma$ or TLR ligands enhances CCL2, CCL5 and CCL20 expression, with no significant changes in CXCL12. Nonetheless, ASC promotion of B-cell chemotaxis does not increase after stimulation. Whereas an inflammatory environment does not modify ASC ability to attract B cells, it might regulate ASC effects on B-cell responses. Other authors found that MSCs can enhance or suppress B-cell antibody secretion, depending on the LPS or viral antigen dose in MSC-B-cell co-cultures (14). Further study is needed to clarify the MSC effects on B-cell responses and the underlying molecular mechanisms.

\section{Acknowledgments}

We thank D. Roy (TiGenix NV) for critical reading of the manuscript, M.A. Iñiguez for advice and help with prostaglandin-related experiments and C. Mark for editorial assistance. LB and VDC were supported by contracts associated with project grants BFU2008-01194 and BFU2011-30097, respectively, from the Spanish Ministry of Economy and Competitiveness (MINECO) to YRC. This work was supported by grants from the Spanish MINECO (BFU2011-30097) to YRC and by funding from the European Union FP7 Programme for research, technological development and demonstration under grant agreement 279174 to TiGenix.

Disclosure of interests: PM, RM, OR, WD and EL are full-time employees of TiGenix. LB, VDC and YRC have no conflicts of interest.

\section{References}

1. Uccelli A, Moretta L, Pistoia V. Mesenchymal stem cells in health and disease. Nat Rev Immunol. 2008;8:726-36.

2. Shi Y, Su J, Roberts AI, Shou P, Rabson AB, Ren G. How mesenchymal stem cells interact with tissue immune responses. Trends Immunol. 2012;33:136-43.

3. Ren G, Su J, Zhang L, Zhao X, Ling W, L'Huillie A, et al. Species variation in the mechanisms of mesenchymal stem cell-mediated immunosuppression. Stem Cells. 2009;27: 1954-62.

4. Duffy MM, Ritter T, Ceredig R, Griffin MD. Mesenchymal stem cell effects on T-cell effector pathways. Stem Cell Res Ther. 2011;2:34.

5. Spaggiari GM, Capobianco A, Abdelrazik H, Becchetti F, Mingari MC, Moretta L. Mesenchymal stem cells inhibit natural killer-cell proliferation, cytotoxicity, and cytokine production: role of indoleamine 2,3-dioxygenase and prostaglandin E2. Blood. 2008;111:1327-33.

6. Spaggiari GM, Abdelrazik H, Becchetti F, Moretta L. MSCs inhibit monocyte-derived DC maturation and function by selectively interfering with the generation of immature DCs: central role of MSC-derived prostaglandin E2. Blood. 2009; 113:6576-83.

7. Chiesa S, Morbelli S, Morando S, Massollo M, Marini C, Bertoni A, et al. Mesenchymal stem cells impair in vivo T-cell priming by dendritic cells. Proc Natl Acad Sci U S A. 2011; 108:17384-9.

8. Francois M, Romieu-Mourez R, Li M, Galipeau J. Human MSC suppression correlates with cytokine induction of 


\section{L. Barrio et al.}

indoleamine 2,3-dioxygenase and bystander M2 macrophage differentiation. Mol Ther. 2012;20:187-95.

9. Edwards JC, Cambridge G. B-cell targeting in rheumatoid arthritis and other autoimmune diseases. Nat Rev Immunol. 2006;6:394-403.

10. Shlomchik MJ. Activating systemic autoimmunity: B's, T's, and tolls. Curr Opin Immunol. 2009;21:626-33.

11. Green NM, Marshak-Rothstein A. Toll-like receptor driven B cell activation in the induction of systemic autoimmunity. Semin Immunol. 2011;23:106-12.

12. Franquesa M, Hoogduijn MJ, Bestard O, Grinyo JM. Immunomodulatory effect of mesenchymal stem cells on B cells. Front Immunol. 2012;3:212.

13. Corcione A, Benvenuto F, Ferretti E, Giunti D, Cappiello V, Cazzanti F, et al. Human mesenchymal stem cells modulate B-cell functions. Blood. 2006;107:367-72.

14. Rasmusson I, Uhlin M, Le Blanc K, Levitsky V. Mesenchymal stem cells fail to trigger effector functions of cytotoxic T lymphocytes. J Leukoc Biol. 2007;82:887-93.

15. Bochev I, Elmadjian G, Kyurkchiev D, Tzvetanov L, Altankova I, Tivchev P, et al. Mesenchymal stem cells from human bone marrow or adipose tissue differently modulate mitogen-stimulated B-cell immunoglobulin production in vitro. Cell Biol Int. 2008;32:384-93.

16. Tabera S, Perez-Simon JA, Diez-Campelo M, SanchezAbarca LI, Blanco B, Lopez A, et al. The effect of mesenchymal stem cells on the viability, proliferation and differentiation of B-lymphocytes. Haematologica. 2008;93:1301-9.

17. Asari S, Itakura S, Ferreri K, Liu CP, Kuroda Y, Kandeel F, et al. Mesenchymal stem cells suppress B-cell terminal differentiation. Exp Hematol. 2009;37:604-15.

18. Schena F, Gambini C, Gregorio A, Mosconi M, Reverberi D, Gattorno M, et al. Interferon-gamma-dependent inhibition of $\mathrm{B}$ cell activation by bone marrow-derived mesenchymal stem cells in a murine model of systemic lupus erythematosus. Arthritis Rheum. 2010;62:2776-86.

19. Lombardo E, DelaRosa O, Mancheno-Corvo P, Menta R, Ramirez C, Buscher D. Toll-like receptor-mediated signaling in human adipose-derived stem cells: implications for immunogenicity and immunosuppressive potential. Tissue Eng Part A. 2009;15:1579-89.

20. Allen CD, Cyster JG. Follicular dendritic cell networks of primary follicles and germinal centers: phenotype and function. Semin Immunol. 2008;20:14-25.

21. Perez-Andres M, Paiva B, Nieto WG, Caraux A, Schmitz A, Almeida J, et al. Human peripheral blood B-cell compartments: a crossroad in B-cell traffic. Cytometry Part B Clin Cytometry. 2010;78(Suppl 1):S47-60.

22. Frade JM, Mellado M, del Real G, Gutierrez-Ramos JC, Lind P, Martinez AC. Characterization of the CCR2 chemokine receptor: functional CCR2 receptor expression in $\mathrm{B}$ cells. J Immunol. 1997;159:5576-84.

23. Zlotnik A, Yoshie O. Chemokines: a new classification system and their role in immunity. Immunity. 2000;12:121-7.

24. Zonca M, Mancheno-Corvo P, DelaRosa O, Manes S, Buscher D, Lombardo E, et al. APRIL and BAFF proteins increase proliferation of human adipose-derived stem cells through activation of Erk1/2 MAP kinase. Tissue Eng Part A. $2012 ; 18: 852-9$

25. Badr G, Borhis G, Lefevre EA, Chaoul N, Deshayes F, Dessirier $\mathrm{V}$, et al. BAFF enhances chemotaxis of primary human B cells: a particular synergy between BAFF and CXCL13 on memory B cells. Blood. 2008;111:2744-54.

26. Yanez R, Oviedo A, Aldea M, Bueren JA, Lamana ML. Prostaglandin E2 plays a key role in the immunosuppressive properties of adipose and bone marrow tissue-derived mesenchymal stromal cells. Exp Cell Res. 2010;316: 3109-23.

27. Kobayashi Y. The role of chemokines in neutrophil biology. Front Biosci. 2008;13:2400-7.

28. Tilley SL, Coffman TM, Koller BH. Mixed messages: modulation of inflammation and immune responses by prostaglandins and thromboxanes. J Clin Invest. 2001;108:15-23.

29. Luft $T$, Jefford $M$, Luetjens $P$, Toy $T$, Hochrein $H$, Masterman KA, et al. Functionally distinct dendritic cell (DC) populations induced by physiologic stimuli: prostaglandin $\mathrm{E}(2)$ regulates the migratory capacity of specific DC subsets. Blood. 2002;100:1362-72.

30. Diaz-Munoz MD, Osma-Garcia IC, Iniguez MA, Fresno M. Cyclooxygenase-2 deficiency in macrophages leads to defective p110gamma PI3K signaling and impairs cell adhesion and migration. J Immunol. 2013;191:395-406.

31. Hoggatt J, Mohammad KS, Singh P, Hoggatt AF, Chitteti BR, Speth JM, et al. Differential stem- and progenitor-cell trafficking by prostaglandin E2. Nature. 2013;495:365-9.

32. Ren G, Zhang L, Zhao X, Xu G, Zhang Y, Roberts AI, et al. Mesenchymal stem cell-mediated immunosuppression occurs via concerted action of chemokines and nitric oxide. Cell Stem Cell. 2008;2:141-50.

33. Rasmusson I, Le Blanc K, Sundberg B, Ringden O. Mesenchymal stem cells stimulate antibody secretion in human B cells. Scand J Immunol. 2007;65:336-43.

34. Nielsen LS, Frydenberg J, Lind M, Deleuran M, StengaardPedersen K, Deleuran B. CD19-selected B lymphocytes synthesize, secrete and migrate in the presence of IL-8. TNFalpha and gammaIP-10 are also B lymphocyte migratory factors. Cytokine. 1997;9:747-53.

35. Jinquan T, Moller B, Storgaard M, Mukaida N, Bonde J, Grunnet N, et al. Chemotaxis and IL-8 receptor expression in B cells from normal and HIV-infected subjects. J Immunol. 1997;158:475-84.

36. Magari M, Nishikawa Y, Fujii Y, Nishio Y, Watanabe K, Fujiwara M, et al. IL-21-dependent B cell death driven by prostaglandin E2, a product secreted from follicular dendritic cells. J Immunol. 2011;187:4210-8.

\section{Supplementary data}

Supplementary data related to this article can be found at http://dx.doi.org/10.1016/j.jcyt.2014.07. 012. 